\title{
Effect of monitored anesthesia care using dexmedetomidine on stress hormones and interleukin-6 in patients undergoing arteriovenous fistula formation
}

\author{
Dong Joon Kim ${ }^{1,2}$, Jeung Wook Park', In Gook Jee ${ }^{1}$, Sang Hun Kim ${ }^{1,2}$ \\ ${ }^{1}$ Department of Anesthesiology and Pain Medicine, Chosun University Hospital, ${ }^{2}$ Department of Anesthesiology and Pain Medicine, School \\ of Medicine, Chosun University, Gwangju, Korea
}

Received February 20, 2018

Revised February 28, 2018

Accepted March 2, 2018

Corresponding author

Sang Hun Kim

Department of Anesthesiology and

Pain Medicine, School of Medicine,

Chosun University, 309 Pilmun-

daero, Dong-gu, Gwangju 61452,

Korea

Tel: $+82-62-220-3223$

Fax: +82-62-223-2333

E-mail: ksh3223@chosun.ac.kr

ORCID:

https://orcid.org/0000-0003-3869-9470

\begin{abstract}
Surgical procedures, as well as anesthetics, affect stress hormones and proinflammatory cytokines. Therefore, we investigated the effect of two different anesthetic techniques on intraoperative hormonal stress responses and interleukin-6 (IL-6) in patients with chronic renal disease undergoing arteriovenous fistula formation. Eighteen patients aged above 20 years were randomly divided into two groups: group $A(n=8)$ with axillary brachial plexus block and group MAC $(n=10)$ with monitored anesthesia care $(M A C)$ using dexmedetomidine. The levels of epinephrine, norepinephrine, cortisol, glucose, IL-6, and heat shock protein 70 (HSP70) were recorded at pre-anesthesia (T0) and end of surgery (T3). No significant differences in epinephrine and HSP70 were observed between these two groups or within each group. Norepinephrine was significantly decreased in group MAC compared with group A at T3 $(p=0.001)$, but no significant differences were found within each group. The cortisol level in group MAC significantly decreased at T3 compared with T0 $(p=0.028)$. The glucose level in group MAC significantly increased at T3 compared with T0 $(p=0.019)$. No significant differences in IL-6 levels were observed within each group. In conclusion, in this study, neither monitored anesthesia nor regional anesthesia influenced stress hormones and proinflammatory cytokines in patients undergoing arteriovenous fistula formation, but significant changes in cortisol and glucose levels were observed in the group receiving dexmedetomidine.
\end{abstract}

Keywords: Brachial plexus block; Dexmedetomidine; Interleukin-6; Chronic kidney failure; Hormones

\section{INTRODUCTION}

Surgery-associated stress response causes extensive immunological changes and can be immunosuppressive [1-3]. Catecholamines and glucocorticoids are the major stress hormones and play a key role in stress-induced suppression of the immune system, and the immune suppression is associ- ated with high risks of infection and postoperative complications [4]. However, recent evidence indicates that the surgeryassociated stress might suppress cellular immunity but boost humoral immunity [5,6]. Stress hormones inhibit the production of proinflammatory cytokines but stimulate the production of anti-inflammatory cytokines [5]. Through modulation of pro/anti-inflammatory cytokine balance, stress hormones

This is an Open Access article distributed under the terms of the Creative Commons Attribution Non-Commercial License (http://creativecommons.org/licenses/by-nc/4.0) which permits unrestricted noncommercial use, distribution, and reproduction in any medium, provided the original work is properly cited. Copyright (c) Medical Biological Science and Engineering. 
may suppress or potentiate the inflammatory response [5]. In addition, one of the interesting stress hormones is extracellular heat shock protein (HSP), which acts as a molecular chaperone and maintains cell homeostasis under physiological and stress conditions [7]. HSP70 has biphasic inflammatory effects: extracellular HSP70 has pro-inflammatory effects, but intracellular HSP70 primarily has anti-inflammatory and antiapoptotic effects [7].

Stress hormones (such as epinephrine, norepinephrine, and cortisol) and proinflammatory cytokines (such as interleukin-6 [IL-6]) can be affected by surgical procedures [8,9], and they also can be influenced by anesthetics and adjuvants during and after anesthesia [10-12]. It was reported that total intravenous anesthesia and regional anesthesia attenuated the increase of stress hormones more than did inhalational anesthesia. However, it is still debated whether the IL-6 level is affected by different anesthetic techniques [10,13]. Furthermore, no reports have compared levels of stress hormones and proinflammatory cytokines during regional anesthesia and monitored anesthesia care (MAC).

Therefore, we hypothesized that MAC with dexmedetomidine and remifentanil attenuates stress-induced changes in hormones and pro-inflammatory cytokines more compared with regional anesthesia with axillary brachial plexus block. The effect of the anesthetic techniques on perioperative changes of stress hormones (a primary outcome) was investigated.

\section{MATERIALS AND METHODS}

This prospective, randomized, controlled, and doubleblinded study was approved by the Institutional Review Board of Chosun University Hospital (IRB no. 11S-263). Written informed consent was obtained from all participants, legal surrogates, or parents or legal guardians of participants who were minors.

A total of 40 patients, who were scheduled to undergo arteriovenous fistula formation because of chronic renal disease, were enrolled in this study. These patients were more than 20 years old and the American Society of Anesthesiologists (ASA) physical status I, II, or III. The patients with preoperative hypertension (above $180 \mathrm{mmHg}$ of systolic blood pressure) or hypotension (below $90 \mathrm{mmHg}$ of systolic blood pressure), preoperative bradycardia (below 50 beats/min), cardiac conduction disorders or arrhythmias, preoperative desaturation (less than 90\%), mental disorders, and allergy or resistance to drugs used in this study; those taking medication comprising other sedatives or anxiolytics; as well as pregnant or breastfeeding women were excluded in this study.

All patients were randomly divided into two groups using a random number table obtained via a computer program (Fig. 1): 1) Group A ( $n=20)$ with regional anesthesia using axillary brachial plexus block and 2) Group MAC ( $\mathrm{n}=20)$ with MAC using dexmedetomidine and remifentanil. Patients and investigators were blinded to the study medications, but a nurse handed out randomized medications (indistinguishable) and numbered syringes using a table of random numbers.

Intramuscular midazolam $(0.05 \mathrm{mg} / \mathrm{kg})$ was administered 30 minutes before anesthesia induction. Subsequently, all patients were transported to the operating room. Before the induction of anesthesia, standard patient monitoring devices were administered. Subsequently, a catheter was installed in a radial artery to continuously monitor blood pressure.

In Group A, an axillary brachial plexus block was performed in position with abduction of the arm $90^{\circ}$ using a 22-gauge, $100 \mathrm{~mm}$ block needle (disposable nerve blockade

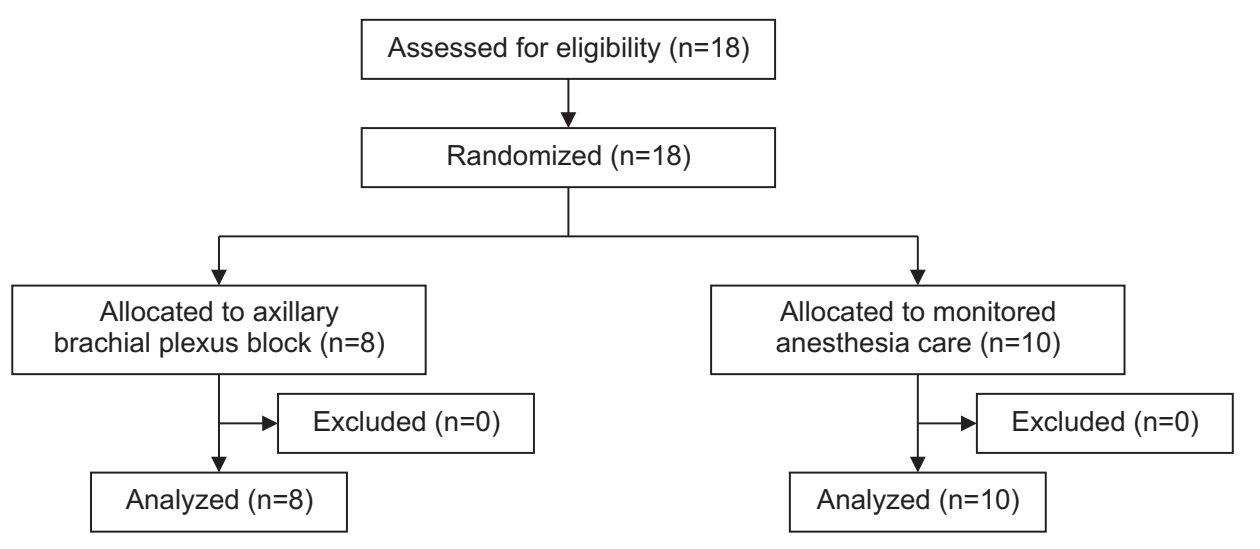

Fig. 1. CONSORT flow chart. 
needle metal hub; Hakko Co., Ltd, Nagano, Japan) and the MTurbo System (SonoSite Inc., Bothwell, WA, USA). In order to prevent the technical variants between performers, all studies were performed by one investigator. After identification of axillary artery and brachial plexus using a 13-6 MHz ultrasound transducer (SonoSite Inc.), the needle was inserted inplane and $0.75 \%$ ropivacaine (each $15 \mathrm{~mL}$ ) was deposited in areas of the radial, ulnar, and median nerve. Finally, the needle was redirected toward the musculocutaneous nerve, and $0.75 \%$ ropivacaine $(10 \mathrm{~mL})$ was deposited. Subsequently, two syringes filled with normal saline were infused using a targetcontrolled infusion (TCI) pump (Orchestra ${ }^{\circledR}$; Fresenius Vial, Brezins, France) with the same techniques as used in group MAC.

In group MAC, an axillary brachial plexus block using normal saline was performed with aforementioned techniques. Thereafter, dexmedetomidine and remifentanil were infused simultaneously. Dexmedetomidine $(1 \mu \mathrm{g} / \mathrm{kg})$ was loaded during 10 minutes, followed by continuous infusion at the infusion rate of $1.0 \mu \mathrm{g} / \mathrm{kg} / \mathrm{h}$ using a TCI pump. Remifentanil was infused at target effect-site concentrations of $2.5 \mathrm{ng} / \mathrm{mL}$ using a TCI pump. Dexmedetomidine and remifentanil concentrations were controlled to maintain the bispectral index score in the range of 40 to 60 and mean arterial pressure changes within $\pm 20 \%$.

Fifteen minutes after anesthesia, surgery started under oxygenation via a facial mask and monitor of $\mathrm{ETCO}_{2}$ so that the proper anesthetic status of each patient was confirmed. If deoxygenation or hypoventilation was happened, the face mask ventilation was administered. If sudden arousal happened, arterial bold gas analysis was performed to evaluate hypercarbia. Systolic hypotension $(\leq 90 \mathrm{mmHg})$ and bradycardia $(\leq 50$ beats $/ \mathrm{min}$ ) were treated with intermittent bolus injections of ephedrine $(10 \mathrm{mg})$ and atropine $(0.5 \mathrm{mg})$, respectively. Systolic hypertension ( $\geq 180 \mathrm{mmHg}$ ) was treated with intermittent bolus injections of perdipine $(1 \mathrm{mg})$.

The levels of epinephrine, norepinephrine, cortisol, glucose, IL-6, and HSP70 were recorded at pre-anesthesia (T0) and end of surgery (T3). The arterial pressure, heart rate, arterial partial pressure of oxygen $\left(\mathrm{PaO}_{2}\right)$, and $\mathrm{ETCO}_{2}$ at fifteen minutes after anesthetic induction (T1), incision (T2), and end of surgery (T3) were compared with those at pre-anesthesia (T0). Age, sex, height, weight, ASA physical status, operation time, and anesthesia time were also recorded.

\section{Statistical analysis}

The necessary sample size was calculated with t-tests of $G^{*}$ Power software (ver. 3.1.9.1) by taking the level of the statistical significance as $\alpha=0.05$ and $\beta=0.2$ using an expected effect size of 0.95 . Total 38 patients were required, but $40 \mathrm{pa}^{-}$ tients were enrolled in this study considering the assumption of a $5 \%$ dropout rate.

IBM SPSS Statistics for Windows ver. 21.0 (IBM Co., Armonk, NY, USA) was used for statistical analysis. All measured values are presented as median (interquartile range), or number of patients. The levels of epinephrine, norepinephrine, cortisol, glucose, IL-6, and HSP70 were analyzed using a MannWhitney $\mathrm{U}$ test between groups and a Wilcoxon signed ranks test within each group because of skewed distribution of data. The changes of arterial pressure, heart rate, $\mathrm{PaO}_{2}$, and $\mathrm{ETCO}_{2}$ were analyzed using a Mann-Whitney $\mathrm{U}$ test between groups because of skewed distribution of data. The sex and ASA physical status were analyzed using the Fisher's exact test. The age, height, weight, operation time, and anesthesia time were analyzed using a Mann-Whitney U test. A p-value less than 0.05 indicates a statistical difference.

\section{RESULTS}

Eighteen patients were ultimately enrolled in this study because of early termination of this study (Fig. 1). No significant differences in sex, age, height, weight, ASA physical status, operation time, and anesthesia time were found between these two groups (Table 1).

The Mann-Whitney U test and Wilcoxon signed rank test indicated that no significant differences in levels of epinephrine and HSP70 were observed between these two groups or within each group at T0 and T3 (Fig. 2).

The level of norepinephrine significantly decreased in group MAC (median: $107.7 \mathrm{pg} / \mathrm{mL}, 22 \mathrm{pg} / \mathrm{mL}$ ) compared with group A (median: $410.4 \mathrm{pg} / \mathrm{mL}, 496.4 \mathrm{pg} / \mathrm{mL}$ ) at T0, T3 (U=8.0, 5.0; $\mathrm{p}=0.003$, 0.001) (Fig. 2). Analysis within each group by a Wilcoxon signed rank test showed that the norepinephrine levels were not significantly different between T3 and T0 (Fig. 2).

The Wilcoxon signed rank test revealed that the levels of cortisol statistically significantly decreased at T3 (median: 9.5 $\mu \mathrm{g} / \mathrm{dL}$ ) compared with T0 (median: $13.2 \mu \mathrm{g} / \mathrm{dL}$ ) in group MAC $(\mathrm{z}=-2.19 ; \mathrm{p}=0.028)$ (Fig. 2). However, no significant differences 
Table 1. Characteristics of patients

\begin{tabular}{lccc}
\hline \multicolumn{1}{c}{ Characteristic } & Group A $(n=8)$ & Group MAC $(n=10)$ & $p-$ value \\
\hline Sex (male/female) & $2 / 6$ & $5 / 5$ & 0.367 \\
ASA physical status (I/II/III) & $0 / 2 / 6$ & $0 / 7 / 3$ & 0.153 \\
Age (y) & $64.0(32.0-68.0)$ & $55.0(44.8-67.8)$ & 0.829 \\
Height $(\mathrm{cm})$ & $158.5(156.0-167.8)$ & $56.5(160.5-170.0)$ & 0.360 \\
Weight $(\mathrm{kg})$ & $58.8(53.5-64.0)$ & $162.5(112.5-197.5)$ & 0.965 \\
Operation time (min) & $152.5(107.5-171.3)$ & $162.5(132.5-213.8)$ & 0.762 \\
Anesthesia time (min) & $180.0(123.8-191.3)$ & 0.633 \\
\hline
\end{tabular}

Values are presented as number only or median (interquartile range). No significant differences were observed between these two groups. $p<0.05$ was considered statistically significant. ASA: American Society of Anesthesiologists.
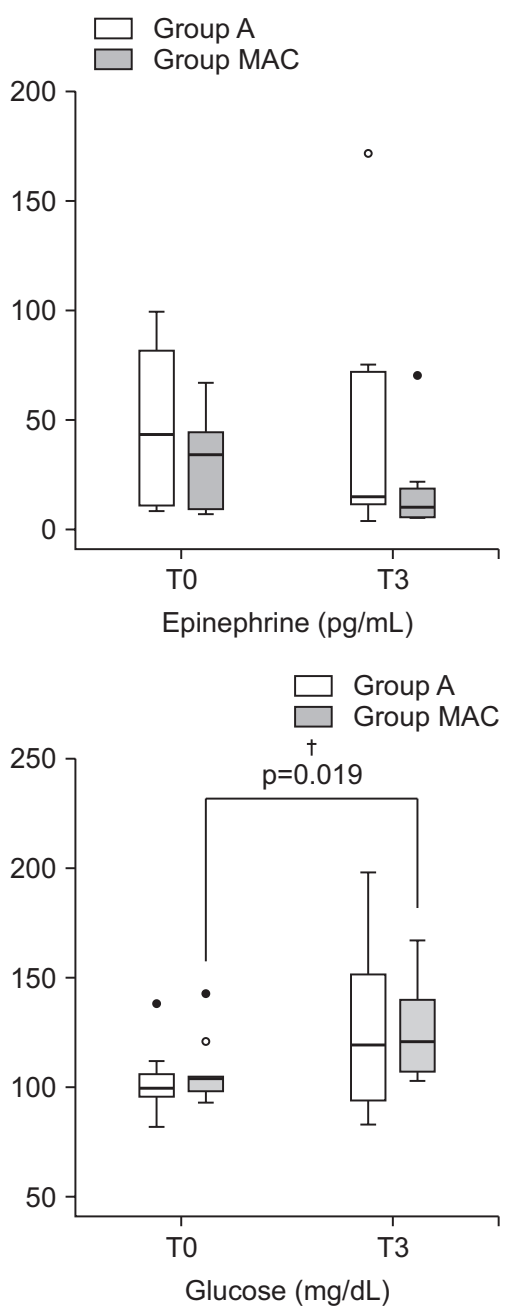
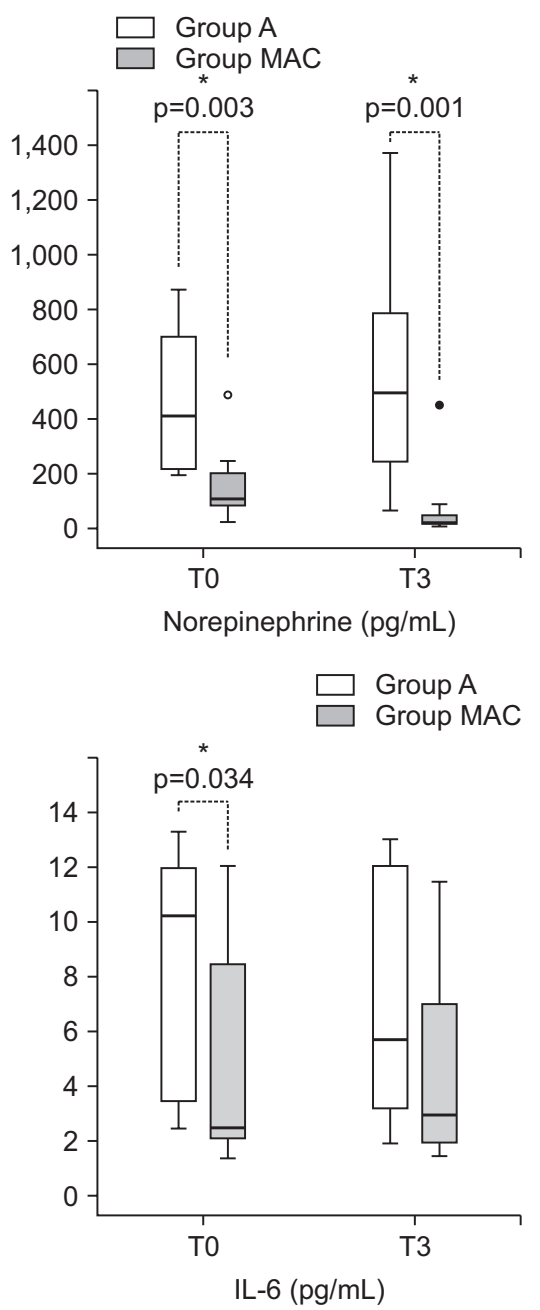
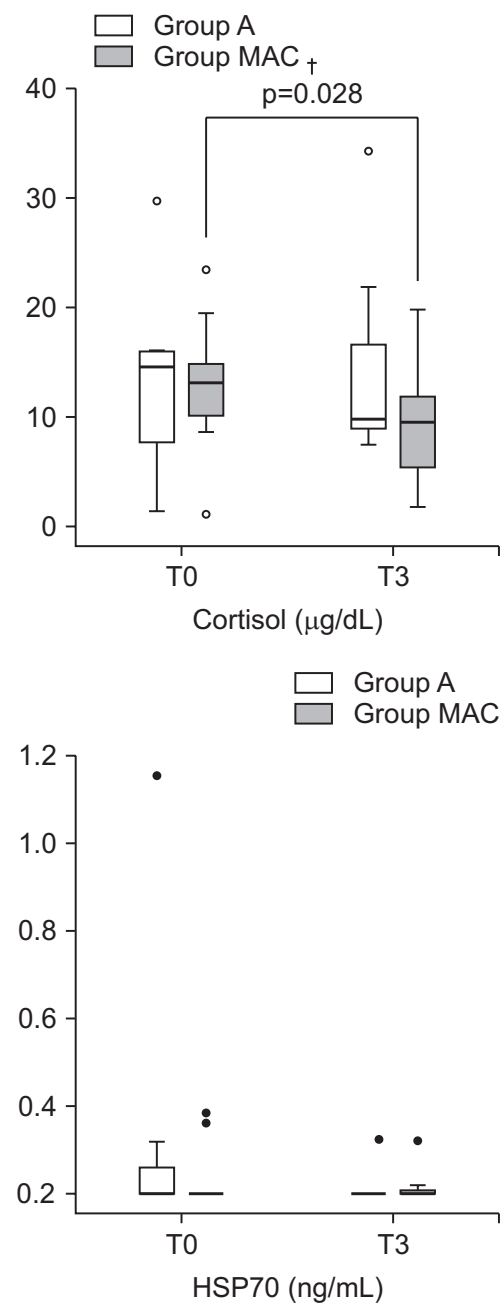

Fig. 2. Levels of epinephrine, norepinephrine, cortisol, glucose, interleukin-6 (IL-6), and heat shock protein 70 (HSP70). Outliers are represented by open circles (०), and extreme outliers are represented by closed circles (•). T0, at pre-anesthesia; T3, at end of surgery. ${ }^{*} p<0.05$ was considered statistically significant when two groups were compared. ${ }^{\dagger} p<0.05$ was considered statistically significant when the values of $\mathrm{T} 0$ and $\mathrm{T} 3$ were compared within each group.

in cortisol levels were observed at T3 compared with $\mathrm{T} 0$ in group A (Fig. 2).

It was found by a Wilcoxon signed rank test that the levels of glucose significantly increased at T3 (median: $121 \mathrm{mg} /$
dL) compared with T0 (median: $104 \mathrm{mg} / \mathrm{dL}$ ) in group MAC $(\mathrm{z}=-2.35 ; \mathrm{p}=0.019)$ (Fig. 2). However, the glucose levels were not significantly different between T3 and T0 in group A (Fig. 2). 




Fig. 3. Intraoperative changes of systolic arterial pressure (SAP), diastolic arterial pressure (DAP), mean arterial pressure (MAP), and heart rate (HR). Outliers are represented by open circles (O), and extreme outliers are represented by closed circles $(\bullet)$. T0, at preanesthesia; T1, at fifteen minutes after anesthetic induction; T2, at incision; $\mathrm{T} 3$, at end of surgery.

The levels of IL-6 significantly decreased in group MAC (median: $2.5 \mathrm{pg} / \mathrm{mL}$ ) compared with group A (median: 10.2 $\mathrm{pg} / \mathrm{mL}$ ) at T0 (U=16; p=0.034) (Fig. 2). Analysis within each group by a Wilcoxon signed rank test showed that the IL-6 levels were not significantly different between $\mathrm{T} 3$ and T0 (Fig. 2).

The levels of arterial pressure, heart rate, $\mathrm{PaO}_{2}$, and $\mathrm{ETCO}_{2}$ were not statistically significantly changed in group MAC compared with group A (Figs. 3 and 4).

\section{DISCUSSION}

This study showed that the cortisol and glucose levels were statistically significantly changed in patients receiving MAC using dexmedetomidine and remifentanil but not in patients receiving regional anesthesia using axillary brachial plexus block at the end of surgery. In addition, the IL-6 level was not significantly altered in both groups.

It has been known that the catecholamine concentration was significantly elevated in patients with chronic renal failure compared with age-matched normal controls, and the concentration of plasma epinephrine and norepinephrine was 77 $\mathrm{pg} / \mathrm{dL}$ and $330 \mathrm{pg} / \mathrm{dL}$, respectively, in patients with renal disease [14,15]. Patients with end-stage renal disease also showed increased levels of pro-inflammatory cytokines (such as IL-1, IL-6, and tumor necrosis factor [TNF]- $\alpha$ ), cortisol, and glucose

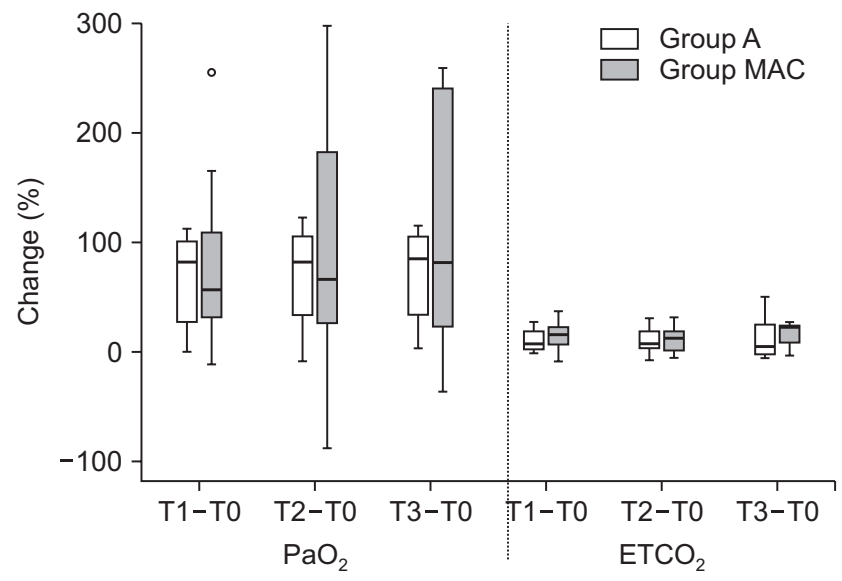

Fig. 4. Intraoperative changes of arterial partial pressure of oxygen $\left(\mathrm{PaO}_{2}\right)$ and end-tidal partial pressure of carbon dioxide $\left(\mathrm{ETCO}_{2}\right)$. Outliers are represented by open circles (०). T0, at pre-anesthesia; T1, at fifteen minutes after anesthetic induction; T2, at incision; T3, at end of surgery.

[16-18]. Particularly, cortisol levels in serum were significantly higher in patients with impaired glomerular filtration rate [19]. In the present study, the baseline values of catecholamines were similar to those in previous published reports, and the baseline values of other parameters were within upper normal ranges in patients with end stage renal disease.

Dexmedetomidine, an $\alpha-2$ adrenoceptor agonist, induces a biphasic cardiovascular response with an initial short-term increased blood pressure followed by a long-lasting decreased blood pressure and heart rate [20]. Dexmedetomidine reduces the circulating catecholamines (such as epinephrine and norepinephrine) on average by approximately $70 \%$, and the role may be associated with its negative inotropic effect [21,22]. In addition, dexmedetomidine significantly decreases the levels of cortisol and glucose, although the levels of catecholamines increase significantly relative to baseline values after surgery, which was aimed to prevent advert hemodynamic changes [23-25]. Dexmedetomidine-induced stress hormonal changes significantly reduce the levels of pro-inflammatory cytokines, such as TNF- $\alpha$, IL-1 $\beta$, and IL- 6 , and stimulate the production of anti-inflammatory cytokines [5,12,26-30].

Patients with chronic renal disease have upregulated HSP70, which might play a role in initiation and/or progression of atherosclerosis [31]. HSP70 protects cell and tissue against stress damage, and particularly, extracellular HSP70 has been reported to have a pro-inflammatory effect [7,32]. Any kind of stress may influence expression of HSP70, but the association of circulating HSP70 with post-surgery complica- 
tions is poorly understood [33,34]. The HSP70 level increased immediately after surgery and was significantly correlated with the IL-6 level [33], and patients with more aggressive surgery had a significantly increased HSP70 level [35].

It has been reported that total intravenous anesthesia attenuates the postoperative increase of cortisol, epinephrine, norepinephrine, and glucose more than does inhalational anesthesia [10,13,36]. Regional anesthesia, such as epidural anesthesia, is also associated with less increased stress hormones levels compared with general anesthesia [11,37]. However, it is still unknown whether the IL- 6 level is affected by different anesthetic techniques [10,13,36]. Furthermore, no reports have compared the levels of stress hormones and pro-inflammatory cytokines between regional anesthesia and MAC.

In this study, no significant changes in catecholamines, cortisol, glucose, IL-6, and HSP70 were observed during regional anesthesia using axillary brachial plexus block. However, the cortisol level significantly decreased, and the glucose level significantly increased in patients receiving MAC using dexmedetomidine, although no significant changes in catecholamines, IL-6, and HSP70 were found.

This discrepancy of results regarding stress hormones, IL6, and HSP70 between previous published reports and our present study may be explained as follows. First, we could not enroll enough patients as planned for the calculated sample size in this study. Thus, this study did not satisfy the initial assumed statistical power (0.8). Analysis of stress hormones, IL6 , and HSP70 had low statistical powers, which were between 0.06 (IL-G) and 0.72 (glucose). Second, the sub-group analysis could not be performed according to the first redo procedure because of low statistical powers. Third, some baseline parameters were significantly different between these two groups because of small sample size, as mentioned above.

In conclusion, in this study, neither monitored anesthesia nor regional anesthesia influenced stress hormones and proinflammatory cytokines in patients undergoing arteriovenous fistula formation, but significant changes in cortisol and glucose levels were found in the group receiving dexmedetomidine. Patients with MAC using dexmedetomidine showed significantly decreased cortisol and increased glucose level at end of surgery compared with before surgery. Therefore, further studies, with increased sample size, are needed for more exact interpretation of this study's results.

\section{ACKNOWLEDGEMENTS}

This study was supported by research funds from Chosun University Hospital 2017.

\section{CONFLICT OF INTEREST}

No potential conflict of interest relevant to this article was reported.

\section{REFERENCES}

1. Munford RS, Pugin J. Normal responses to injury prevent systemic inflammation and can be immunosuppressive. Am J Respir Crit Care Med 2001;163:316-21.

2. Isitmangil G, Isitmangil T, Balkanli K, Cerrahoglu K, Kunter E. Detection of thoracotomy-induced alterations in cell- and humoral-mediated immune response. Eur J Cardiothorac Surg 2002;21:497-501.

3. O'Sullivan ST, Lederer JA, Horgan AF, Chin DH, Mannick JA, Rodrick ML. Major injury leads to predominance of the $\mathrm{T}$ helper-2 lymphocyte phenotype and diminished interleukin-12 production associated with decreased resistance to infection. Ann Surg 1995;222:482-90; discussion 490-2.

4. Ahlers O, Hildebrandt B, Dieing A, Deja M, Böhnke T, Wust P, et al. Stress induced changes in lymphocyte subpopulations and associated cytokines during whole body hyperthermia of 41.842.2 degrees C. Eur J Appl Physiol 2005;95:298-306.

5. Elenkov IJ, Chrousos GP. Stress hormones, proinflammatory and antiinflammatory cytokines, and autoimmunity. Ann N Y Acad Sci 2002;966:290-303.

6. Elenkov IJ, Chrousos GP. Stress hormones, Th1/Th2 patterns, pro/anti-inflammatory cytokines and susceptibility to disease. Trends Endocrinol Metab 1999;10:359-68.

7. Huang F, Huang JP, Pan JY, Bai ZL, Tang L, Zhou SH. Intracellular heat shock protein 70: a possible therapeutic target for preventing postoperative atrial fibrillation. Pharmazie 2012; 67:747-55.

8. Roth-Isigkeit A, Brechmann J, Dibbelt L, Sievers HH, Raasch W, Schmucker P. Persistent endocrine stress response in patients undergoing cardiac surgery. J Endocrinol Invest 1998;21:12-9.

9. Hoda MR, El-Achkar H, Schmitz E, Scheffold T, Vetter HO, De Simone R. Systemic stress hormone response in patients undergoing open heart surgery with or without cardiopulmonary bypass. Ann Thorac Surg 2006;82:2179-86.

10. Schneemilch CE, Bank U. Release of pro- and anti-inflammatory cytokines during different anesthesia procedures. Anaesthesiol Reanim 2001;26:4-10.

11. Yokoyama M, Itano Y, Mizobuchi S, Nakatsuka H, Kaku R, Takashima T, et al. The effects of epidural block on the distri- 
bution of lymphocyte subsets and natural-killer cell activity in patients with and without pain. Anesth Analg 2001;92:463-9.

12. Venn RM, Bryant A, Hall GM, Grounds RM. Effects of dexmedetomidine on adrenocortical function, and the cardiovascular, endocrine and inflammatory responses in post-operative patients needing sedation in the intensive care unit. Br J Anaesth 2001;86:650-6.

13. Ihn CH, Joo JD, Choi JW, Kim DW, Jeon YS, Kim YS, et al. Comparison of stress hormone response, interleukin-6 and anaesthetic characteristics of two anaesthetic techniques: volatile induction and maintenance of anaesthesia using sevoflurane versus total intravenous anaesthesia using propofol and remifentanil. J Int Med Res 2009;37:1760-71.

14. Darwish R, Elias AN, Vaziri ND, Pahl M, Powers D, Stokes JD. Plasma and urinary catecholamines and their metabolites in chronic renal failure. Arch Intern Med 1984;144:69-71.

15. Laederach K, Weidmann P. Plasma and urinary catecholamines as related to renal function in man. Kidney Int 1987;31: 107-11.

16. Rios DRA, Pinheiro MB, de Oliveira Junior WV, Braga Gomes $\mathrm{K}$, Carvalho AT, Martins-Filho OA, et al. Cytokine signature in end-stage renal disease patients on hemodialysis. Dis Markers 2017;2017:9678391.

17. Lam CW. 2. Inflammation, cytokines and chemokines in chronic kidney disease. EJIFCC 2009;20:12-20.

18. Slee AD. Exploring metabolic dysfunction in chronic kidney disease. Nutr Metab (Lond) 2012;9:36.

19. Li X, Xiang X, Hu J, Goswami R, Yang S, Zhang A, et al. Association between serum cortisol and chronic kidney disease in patients with essential hypertension. Kidney Blood Press Res 2016;41:384-91.

20. Link RE, Desai K, Hein L, Stevens ME, Chruscinski A, Bernstein D, et al. Cardiovascular regulation in mice lacking alpha2adrenergic receptor subtypes b and c. Science 1996;273:803-5.

21. Talke P, Li J, Jain U, Leung J, Drasner K, Hollenberg M, et al. Effects of perioperative dexmedetomidine infusion in patients undergoing vascular surgery. The Study of Perioperative Ischemia Research Group. Anesthesiology 1995;82:620-33.

22. Snapir A, Posti J, Kentala E, Koskenvuo J, Sundell J, Tuunanen $\mathrm{H}$, et al. Effects of low and high plasma concentrations of dexmedetomidine on myocardial perfusion and cardiac function in healthy male subjects. Anesthesiology 2006;105:902-10; quiz 1069-70.

23. El-Tahan MR, Mowafi HA, Al Sheikh IH, Khidr AM, Al-Juhaiman RA. Efficacy of dexmedetomidine in suppressing cardiovascular and hormonal responses to general anaesthesia for caesarean delivery: a dose-response study. Int J Obstet Anesth 2012;21:222-9.

24. Uyar AS, Yagmurdur H, Fidan Y, Topkaya C, Basar H. Dexmedetomidine attenuates the hemodynamic and neuroendocrinal responses to skull-pin head-holder application during crani- otomy. J Neurosurg Anesthesiol 2008;20:174-9.

25. Bekker A, Haile M, Kline R, Didehvar S, Babu R, Martiniuk F, et al. The effect of intraoperative infusion of dexmedetomidine on the quality of recovery after major spinal surgery. J Neurosurg Anesthesiol 2013;25:16-24.

26. Can M, Gul S, Bektas S, Hanci V, Acikgoz S. Effects of dexmedetomidine or methylprednisolone on inflammatory responses in spinal cord injury. Acta Anaesthesiol Scand 2009;53:1068-72.

27. Kang SH, Kim YS, Hong TH, Chae MS, Cho ML, Her YM, et al. Effects of dexmedetomidine on inflammatory responses in patients undergoing laparoscopic cholecystectomy. Acta Anaesthesiol Scand 2013;57:480-7.

28. Hofer S, Steppan J, Wagner T, Funke B, Lichtenstern C, Martin E, et al. Central sympatholytics prolong survival in experimental sepsis. Crit Care 2009;13:R11.

29. Memiş D, Hekimoğlu S, Vatan I, Yandim T, Yüksel M, Süt N. Effects of midazolam and dexmedetomidine on inflammatory responses and gastric intramucosal $\mathrm{pH}$ to sepsis, in critically ill patients. Br J Anaesth 2007;98:550-2.

30. Tasdogan M, Memis D, Sut N, Yuksel M. Results of a pilot study on the effects of propofol and dexmedetomidine on inflammatory responses and intraabdominal pressure in severe sepsis. J Clin Anesth 2009;21:394-400.

31. Yadav AK, Kumar V, Jha V. Heat shock proteins 60 and 70 specific proinflammatory and cytotoxic response of CD4+CD28null cells in chronic kidney disease. Mediators Inflamm 2013;2013:384807.

32. Honsawek S, Udomsinprasert W, Jirathanathornnukul N, Chongsrisawat V, Poovorawan Y. Elevated serum heat shock protein 70 and liver stiffness reflect hepatic dysfunction and severity in postoperative biliary atresia. Pediatr Surg Int 2017; 33:893-9.

33. Kimura F, Itoh H, Ambiru S, Shimizu H, Togawa A, Yoshidome $\mathrm{H}$, et al. Circulating heat-shock protein 70 is associated with postoperative infection and organ dysfunction after liver resection. Am J Surg 2004;187:777-84.

34. Yu H, Bao ED, Zhao RQ, Lv QX. Effect of transportation stress on heat shock protein 70 concentration and mRNA expression in heart and kidney tissues and serum enzyme activities and hormone concentrations of pigs. Am J Vet Res 2007;68:1145-50.

35. Gülnahar Y, Hüseyin Köşger H, Tutar Y. A comparison of piezosurgery and conventional surgery by heat shock protein 70 expression. Int J Oral Maxillofac Surg 2013;42:508-10.

36. Kostopanagiotou G, Kalimeris K, Christodoulaki K, Nastos C, Papoutsidakis N, Dima C, et al. The differential impact of volatile and intravenous anaesthetics on stress response in the swine. Hormones (Athens) 2010;9:67-75.

37. Milosavljevic SB, Pavlovic AP, Trpkovic SV, Ilić AN, Sekulic AD. Influence of spinal and general anesthesia on the metabolic, hormonal, and hemodynamic response in elective surgical patients. Med Sci Monit 2014;20:1833-40. 